Original

\title{
Estudio comparativo entre la eficacia analgésica y seguridad de la meperidina intravenosa frente a la infiltración periprostática con lidocaina en biopsias transrectales de próstata
}

\author{
Sátur Luján Marco, Vanessa Bango García, Salvador Arlandis Guzmán, Carlos Di Capua Sacoto, \\ Pilar Bahílo Mateu, Marta Trassierra Villa, Miguel Ramírez Backhaus, Juan F. Jiménez Cruz
}

Servicio de Urología. Hospital Universitario La Fe Valencia.

\begin{abstract}
Resumen
Objetivo: Evaluar la eficacia de la analgesia intravenosa con meperidina frente a la infiltración del plexo periprostático con lidocaina,y seguridad de la anestesia local periprostática.

Material y métodos: Estudio prospectivo aleatorizado con 100 pacientes sometidos a primera o segunda biopsia de próstata. Distribuimos a los pacientes en dos grupos; grupo A (50 pacientes) al que se le administra $50 \mathrm{mg}$ de meperidina intravenosa y grupo B (50 pacientes) que reciben $5 \mathrm{~mL}$ de lidocaína al $2 \%$ en el ángulo prostatoseminal. El dolor fue evaluado mediante la Escala Analógico Visual (VAS) y un cuestionario a cerca del impacto emocional de la prueba. La seguridad del procedimiento se obtuvo mediante cuestionario telefónico sobre las complicaciones aparecidas tras la biopsia. Para el análisis estadístico se uso el test de chi cuadrado, la t de Student y test no paramétrico de Kruskal-Wallis.

Resultados: La mediana de edad fue 66 años (47-80) siendo ambos grupos homogéneos respecto a: PSA, volumen prostático, número de cilindros y nivel de estudios, sin diferencias significativas. Se trató de una primera biopsia en 74 pacientes (74\%) y 26 (26\%)

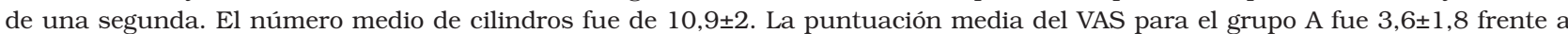
$3,2 \pm 2$ del grupo B sin diferencias significativas $(p>0,05)$. Si comparamos el dolor con la introducción del transductor (grupo A $3,9 \pm 1,9$ / grupo B 4,3 $\pm 2,2$ ) frente a la toma de cilindro (grupo A 3,6 $\pm 1,8$ /grupo B 3,2 \pm 22 ) sí encontramos diferencias significativas $(p<0,05)$. No se observan diferencias ente los datos obtenidos con el test de impacto emocional, la edad y el nivel de estudios frente al dolor producido por la prueba. Respecto al número de cilindros obtenidos tampoco existen diferencias (p>0,05). Aparecieron complicaciones en 12 pacientes (12\%); 5 en el grupo de meperidina frente a 7 en el de lidocaína sin observar diferencias entre ambos.

Conclusión: El bloqueo del plexo periprostático con lidocaína no ofrece ventajas analgésicas respecto a la meperidina, a pesar de que se trata de un método seguro que no aumenta el número de complicaciones. El paso del transductor transrrectal produce más dolor que la propia toma de los cilindros.
\end{abstract}

Palabras clave: Biopsia de Próstata. Meperidina. Infiltración periprostática. Lidocaína.

\section{Comparative study between analgesic efficacy and safety of meperidine compared with lidocaine periprostatic infiltration in transrectal ultrasound guided prostate biopsy}

\section{Abstract}

Objectives: To assess the efficacy of intravenous analgesia with meperidine compared to periprostatic plexus infiltration with lidocaine, and safety of periprostatic local anesthesia.

Materials and methods: A prospective randomized study with 100 patients undergoing first or second prostate biopsy. We distribute patients in two groups, group A (50 patients) which was administered $50 \mathrm{mg}$ of intravenous meperidine and group B (50 patients) receiving $5 \mathrm{~mL}$ of lidocaine $2 \%$ in the angle between prostate and seminal vesicles. Pain was assessed by Visual Analog Scale (VAS) and a questionnaire about the emotional impact. Procedure safety was obtained by telephone questionnaire about prostate biopsy complications. The statistical analysis used was chi square test, Student's test and Kruskal-Wallis no parametric test. Results: Median age was 66 years (47-80) and both groups were homogeneous with regard to: PSA, prostate volume, core's number and educational level without significant differences. 74 patients (74\%) had their first biopsy and 26 (26\%) had their second one. The average number of core biopsy was $10,9 \pm 2$, and VAS mean score for group A was 3,6 $\pm 1,8$ versus $3,2 \pm 2$ Group B without significant differences $(\mathrm{p}>0,05)$. We found significant differences $(\mathrm{p}<0,05)$ between transducer introduction $(3,9 \pm 1,9$ group $\mathrm{A} / \mathrm{B}$ group $4,3 \pm 2,2)$ and core biopsy $(3,6 \pm 1,8$ group A/B group $3,2 \pm 2,2)$. There were no differences between the data obtained with emotional impact test, age and educational level comparing to pain caused by prostate biopsy. Regard to the number of cores obtained there were no differences $(\mathrm{p}>0,05)$. Complications appeared in 12 patients $(12 \%), 5$ in the group of meperidine compared with 7 in the lidocaine without differences between them.

Conclusion: Periprostatic plexus blocked with lidocaine does not offer advantages respect to meperidine, despite the fact that this is a safe method that does not increase the number of complications. Placing transrectal transducer causes more pain than biopsy cores.

Keywords: Prostate biopsy. Meperidine. Periprostatic infiltration. Lidocaine. 
$\mathrm{L}^{2}$ a biopsia prostática ecodirigida es una práctica habitual en todos los hospitales, como principal prueba diagnóstica del cáncer de próstata. Existen discrepancias acerca de sus indicaciones, el número de cilindros que debemos tomar y la preparación del paciente ${ }^{1}$, así como los métodos analgésicosanestésicos a emplear. Entre un 7 y un $25 \%$ de los pacientes biopsiados refieren dolor moderado o intenso y hasta un $20 \%$ no volverían a someterse de nuevo a esta técnica ${ }^{2,3}$. Por tanto, teniendo en cuenta que la biopsia prostática es un método diagnóstico cruento unido a la preocupación del paciente ante la posibilidad de padecer cáncer provocan que esta sea una técnica "traumática” para los pacientes. Nuestro objetivo es disminuir dicha ansiedad con una información adecuada y ofrecer la mejor analgesia disponible al paciente. En los últimos años la tendencia ha sido aumentar el número de cilindros que se toman durante la biopsia prostática, con el fin de incrementar su rendimiento diagnóstico. A mayor número de cilindros, mayores son las molestias producidas al paciente ${ }^{4}$, por eso nos hemos planteado en este estudio, encontrar una alternativa anestésica a la meperidina (50 mg) que clásicamente veníamos utilizando en nuestro servicio con el fin de disminuir el dolor. La utilización de la lidocaína como agente anestésico en biopsias de próstata fue descrito por primera vez por Nash et al. ${ }^{5}$, desde entonces, son numerosos los trabajos publicados que hablan de la eficacia y seguridad de este tipo de técnica.

\section{MATERIAL Y MÉTODOS}

Realizamos un estudio prospectivo y aleatorizado en el que se han incluido a 100 pacientes que han sido sometidos a primera o segunda biopsia de próstata en nuestro hospital durante un periodo de 6 meses. La indicación de biopsia prostática fue el hallazgo de un PSA mayor de $10 \mathrm{ng} / \mathrm{ml}$, o entre 4$10 \mathrm{ng} / \mathrm{ml}$ con PSA Libre/total por debajo del 20\% y/o un tacto rectal sospechoso.

El número de cilindros a obtener durante la biopsia fue de 10 muestras en caso de primera biopsia y 14 muestras en el caso de una segunda biopsia. Excluimos del estudio a pacientes sometidos a terceras biopsias y posteriores, en los que utilizamos ambos métodos (meperidina intravenosa más anestesia del plexo), por considerar el procedimiento más incómodo y doloroso para el paciente, al aumentar el número de cilindros a tomar.
Distribuimos a los pacientes en dos grupos (A y B), cuya aleatorización se estableció en días alternos: Grupo A, que incluyó 50 pacientes (50\%) a los que se administró $50 \mathrm{mg}$ de meperidina intravenosa 30 minutos antes de la biopsia; Grupo B, que incluyó otros 50 pacientes (50\%) a los que se les infiltró $5 \mathrm{ml}$ de lidocaína al $2 \%$ a nivel del ángulo prostatoseminal.

Se realizó una preparación intestinal previa mediante la aplicación de un enema de limpieza la noche antes de la biopsia y la misma mañana de la prueba. Como profilaxis antibiótica administramos $100 \mathrm{mg}$ de tobramicina endovenosa 30 minutos antes de la biopsia, seguida de ciprofloxacino $500 \mathrm{mg}$ cada 12 horas vía oral durante 72 horas. Para el procedimiento usamos el ecógrafo Siemens Sonoline Antares ${ }^{\circledR}$ monoplano de $7,5 \mathrm{mHz}$. Una vez introducido el transductor, al grupo B se les administró $5 \mathrm{ml}$ de lidocaína al $2 \%$ con una aguja Chiba de $22 \mathrm{G}$, en un único punto de punción. La región de la próstata que en nuestro caso identificamos como el lugar óptimo de punción, se encuentra entre la cápsula prostática y la fascia de Denonvilliers, en el punto donde ambas vesículas seminales se unen a la próstata, fácilmente identificable mediante ecografía transrectal, y caracterizada por ser una zona intensamente hiperecogénica que se conoce como "signo del Everest" (Fig. 1).

Se toman las muestras con un dispositivo de resorte con una aguja de punción $18 \mathrm{G}$, que obtiene cilindros de $25 \mathrm{~mm}$ de longitud y aproximadamente $1 \mathrm{~mm}$ de grosor.

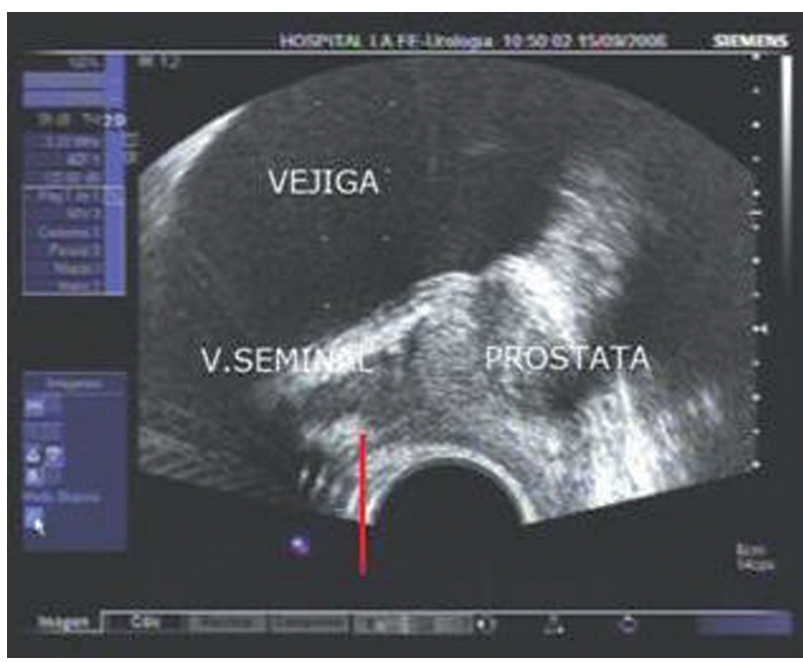

FIGURA 1. Punto de punción (línea roja) caracterizado por ser una zona hiperecogénica entre vesícula y próstata conocida como "signo del Everest". 
Para evaluar la eficacia del método utilizado, el paciente contestó un cuestionario compuesto por una serie de preguntas acerca del impacto emocional que suponía el procedimiento (Fig. 2).

- ¿Creee usted que es una persona...?
1. Tranquila
2. Nerviosa
3. Importante

- ¿Qué grado de nerviosismo tenía antes de la prueba?
1. Ninguno
2. Regular
3. Importante

- ¿Qué preocupación tiene ante el resultado de la prueba?
1. Ninguna
2. Regular
3. Importante

- La información que ha recibido antes de la prueba sobre ella, le parece...
1. Insuficiente
2. Suficiente 3. Abundante

- Antes de hacerse la prueba pensaba que sería: 1. Nada molesta 2. Molesta 3. Dolorosa

FIGURA 2. Test de impacto emocional.

Además, se recogieron datos de tipo socioeconómico, como el nivel de estudios y la profesión del paciente, así como la edad, número de biopsias de próstata, número de cilindros obtenidos, valor del PSA y el volumen prostático obtenido por ecografía transrectal.

El dolor se evaluó mediante una Escala Analógico Visual (VAS), diferenciando dos fases en el procedimiento: VAS1, que cuantifica el dolor producido por la introducción del transductor transrrectal; y VAS2 que mide el dolor producido por la toma de cilindros prostáticos (Fig. 3). Según esta escala, 0 correspondería a nada de dolor, 1,2 y 3 corresponderían a un dolor leve; 4,5 y 6 a un dolor moderado; 7,8 y 9 a un dolor severo y 10 al peor dolor posible.

La seguridad del procedimiento se evaluó mediante encuesta telefónica y revisión de historias clínicas recogiendo datos acerca de las complicaciones aparecidas tras la biopsia (rectorragia, hematuria, uretrorragia, hemospermia, retención aguda de orina o fiebre).

\section{$\begin{array}{lllllllllll}0 & 1 & 2 & 3 & 4 & 5 & 6 & 7 & 8 & 9 & 10\end{array}$} NADA DE DOLOR FIGURA 3. Escala analógico visual administrada a los pacientes. Se valoraron 2 momentos; VAS1, introducción del transductor y VAS2, toma de cilindros.
Analizamos los valores obtenidos en la VAS en ambos grupos mediante un test estadístico de comparación de medias (t de Student para muestras independientes) e intentamos establecer una relación entre la edad y el dolor provocado al paciente. Mediante el test no paramétrico de Kruskal-Wallis, se analiza la relación que puede existir entre los resultados obtenidos en la VAS y las preguntas administradas al paciente (test de impacto emocional) (Fig. 1), así como el efecto que el nivel de estudios del paciente pudiera causar en la percepción del dolor. Con el test de Chi cuadrado, analizamos si la anestesia del plexo periprostático aumenta las complicaciones de la biopsia prostática.

\section{RESULTADOS}

La mediana de edad fue de 66 años (47-80) y el nivel medio de estudios en nuestra muestra correspondió a un nivel medio. Ambos grupos fueron homogéneos (Tabla 1) en cuanto a los valores de PSA, tamaño prostático, edad, número de cilindros obtenidos durante la biopsia, y el nivel de estudios, no existiendo diferencias estadísticamente significativas entre ellos $(\mathrm{p}>0,05)$.

Tabla 1: Valores medios de PSA, volumen prostático, número de cilindros y edad de los pacientes. Ambos grupos fueron homogéneos sin que existieran diferencias estadísticas ( $p>0,05)$

\begin{tabular}{ccccc}
\hline & $\begin{array}{c}\text { PSA } \\
\text { media } \pm \text { DS }\end{array}$ & $\begin{array}{c}\text { Vol } \\
\text { media } \pm \text { DS }\end{array}$ & $\begin{array}{c}\text { Cilindros } \\
\text { media } \pm \text { DS }\end{array}$ & $\begin{array}{c}\text { Edad } \\
\text { media } \pm D S\end{array}$ \\
\hline Grupo A & $10 \pm 15$ & $51 \pm 22$ & $10,9 \pm 2,2$ & $64 \pm 9,5$ \\
Grupo B & $11 \pm 20$ & $51 \pm 28$ & $10,9 \pm 1,8$ & $65 \pm 6,5$ \\
\hline
\end{tabular}

Un total de 74 pacientes (74\%), se sometieron por primera vez a una biopsia de próstata y 26 (26\%) lo hicieron por segunda vez. El número medio de cilin-

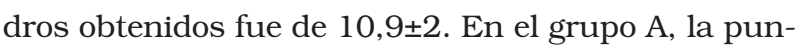
tuación media del VAS fue de $3,6 \pm 1,8$ y en el grupo $B$, la media fue $3,2 \pm 2$, sin que existan diferencias estadísticamente significativas entre ambos grupos $(\mathrm{p}=0,39)$. Si diferenciamos la introducción del transductor (VAS 1), de la propia toma de muestras (VAS 2), observamos que la media del VAS1 en ambos grupos es de $4,1 \pm 2,1$, frente a $3,4 \pm 1,9$ del VAS2, con diferencias estadísticamente significativas $(p=0,04)$. Respecto a la influencia de la edad y teniendo en cuenta la distribución homogénea de los pacientes dividimos en dos segmentos: los de edad inferior o igual a 65 y los mayores de 65 (Tabla 2). No existen 
diferencias estadísticamente significativas entre la edad de los pacientes y los resultados obtenidos en la VAS independientemente del método analgésico utilizado ( $>>0,05)$.

En cuanto a la influencia del número de cilindros y el dolor dividimos a los pacientes en dos grupos. Un primer grupo correspondería a aquellos pacientes en los que se han obtenido 12 o menos muestras (VAS 2=3,5 $\pm 1,99$ ), y un segundo grupo en

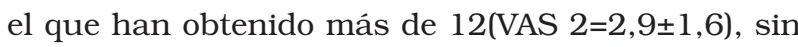
apreciar diferencias significativas $(p=0,14)$ (Tabla 3).

Tabla 2: Diferenciamos dos grupos de edad; mayor de 65 años y menor o igual a 65 y calculamos la puntuacón media del dolor en dos momentos de la biopsia sin que existan diferencias significativas $(\mathrm{p}>0,05)$.

\begin{tabular}{lcc}
\hline Edad & $\begin{array}{c}\text { VAS1 } \\
\text { media } \pm \text { DS }\end{array}$ & $\begin{array}{c}\text { VAS2 } \\
\text { media } \pm D S\end{array}$ \\
\hline$\leq 65$ & $4,16 \pm 1,9$ & $3,53 \pm 2$ \\
$>65$ & $4,12 \pm 2,2$ & $3,35 \pm 1,8$ \\
\hline
\end{tabular}

Tabla 3: La puntuación del dolor en función del número de cilindros, mayor de 12 frente a menor o igual a 12, varia entre ambos grupos sin que existan diferencias estadísticas (p 0,14).

\begin{tabular}{cc}
\hline $\mathbf{N}^{\circ}$ cilindros & $\begin{array}{c}\text { VAS2 } \\
\text { media } \pm \text { DS }\end{array}$ \\
\hline$\leq 12$ & $3,5 \pm 1,99$ \\
$>12$ & $2,9 \pm 1,6$ \\
\hline
\end{tabular}

Por otro lado analizamos la probable relación entre el dolor producido por la biopsia mediante los resultados en la VAS 1 y 2 , con la personalidad del paciente, nerviosismo previo a la prueba, grado de preocupación por el resultado de la misma así como el nivel de estudios, obtenidos mediante el test de impacto emocional. No encontramos diferencias significativas entre el dolor y estas variables $(p>0,05)$.

Del total de 100 pacientes, aparecieron 12 complicaciones (12\%); 5 en el grupo que recibió meperidina y 7 en el grupo que recibió lidocaína, sin que existan diferencias estadísticamente significativas entre ambos grupos $(p=0,75)$. La complicación más frecuente fue la fiebre, que apareció en 6 pacientes (6\%), 3 casos (3\%) en el grupo A y 3 casos (3\%) en el grupo B. Se dieron 2 casos (2\%) de rectorragia, uno de ellos que requirió tratamiento mediante esclerosis del punto sangrante, 2 casos $(2 \%)$ de retención aguda de orina, y $2(2 \%)$ de hematuria franca.

\section{DISCUSIÓN}

Aunque la biopsia prostática ecodirigida es considerada como una prueba bien tolerada y con escasas complicaciones, hoy en día queda claro que se trata de una prueba cuando menos molesta, y que algunos pacientes llegan a definir como insoportable.

Los principales factores responsables del dolor son la introducción del transductor por vía transrrectal y la toma de cilindros prostáticos, si bien en la apreciación del dolor también influyen otros factores como el miedo al diagnóstico de cáncer, la edad del paciente, el grado de ansiedad previo a la biopsia y la propia vía de obtención de muestras que para algunos pacientes supone una experiencia traumática. Bastide et al. ${ }^{6}$ proponen identificar grupos de riesgo dentro de los pacientes con el fin de seleccionar aquellos que podrían beneficiarse más de una técnica anestésica, si bien en nuestro estudio no hemos encontramos relación entre los datos obtenidos mediante el test de impacto emocional (personalidad del paciente, nerviosismo antes de la prueba, etc.) y los resultados en la VAS 1 y 2 , tal y como observan otros autores ${ }^{7}$. Tampoco hemos objetivado diferencias significativas en la valoración del dolor en función del nivel cultural y la edad del paciente, al contrario que en el estudio de Rodríguez et al. ${ }^{8}$.

Nash et al. ${ }^{5}$ realizaron la primera descripción del bloqueo de haces neurovasculares con lidocaina. Desde entonces, han sido muchos los artículos publicados en los que se califica a esta técnica como el "gold standard" cuando se compara con gel de lidocaína intrarrectal y placebo ${ }^{9}$ aunque existen estudios donde no se muestran dichas diferencias ${ }^{10}$.

No hay en la actualidad un consenso respecto a la dosis de fármaco a utilizar y sobre todo, el lugar óptimo de punción: intracapsular, periprostático apical o ángulo vesicoprostático, así como inyección única o bilateral, etc. Según nuestros resultados, el bloqueo del plexo periprostático con lidocaína no es más efectivo que la meperidina intravenosa. Tampoco se han encontraron diferencias cuando se compara la anestesia del plexo periprostático con un analgésico opioide como el tramadol ${ }^{11}$.

Una de las razones por las cuales la efectividad sea similar pueda ser debido a la necesidad de infiltrar el anestésico, no sólo en un único punto de punción, sino a ambos lados de la próstata, como defienden otros autores. En este estudio nos decan- 
tamos por un único punto de punción por considerarlo más inocuo y cómodo para el paciente que instilar anestésico bilateralmente, como defiende Taverna et al. ${ }^{12}$.

Un dato llamativo que también muestran otros estudios $^{5}$, es el hecho de la introducción de la sonda transrectal y los movimientos de ésta durante la biopsia le producen al paciente más incomodidad que propia toma de cilindros prostáticos. Esto subraya la conveniencia de administrar algún gel anestésico intrarrectal (gel de lidocaína) ${ }^{13,14}$, previo a la introducción del transductor con infiltración posterior del plexo periprostático, o bien conseguir una dilatación anal lenta y progresiva antes de insertar el transductor.

A medida que el número de cilindros obtenidos durante la biopsia aumentan, también el dolor lo hace $^{4}$. La biopsia sextante, independientemente del método analgésico que se utilice, es mejor tolerada que la toma de 10 o más muestras. El dolor parece tener cierto carácter acumulativo, según muestran algunos estudios ${ }^{15}$, sin embargo hemos podido observar que paradójicamente una mayor número de cilindros (superior a 12) puntúa menos en la escala analógico visual que una menor toma de muestras (menor de 12) sin observar diferencias significativas.

Las complicaciones más frecuentes derivadas de la biopsia prostática son la uretrorragia, rectorragia y fiebre. El sangrado parece estar más relacionado con el número de muestras obtenidas durante la biopsia y la localización de éstas (cuanto más cerca de la uretra, más riesgo de sangrado). Dado que la infiltración del plexo periprostático requiere de al menos un pinchazo extra, cabe esperar que aumente la incidencia de complicaciones, pero en varios trabajos no se observan diferencias significativas. En nuestro estudio no hemos encontrado un aumento en la incidencia de fiebres post-biopsia cuando mayor ha sido el número de cilindros obtenidos. Obek et $\mathrm{al}^{16}$, observaron que la administración de lidocaína lograba disminuir la frecuencia de rectorragias.

No existen discrepancias a cerca de la necesidad de utilizar algún tipo de analgesia que disminuya las molestias del paciente, por ello, debemos encontrar la opción más efectiva y segura. La meperidina intravenosa es un sistema rápido, barato, fácil de administrar y con pocas contraindicaciones de uso. No hemos observado efectos secundarios derivados de su uso, por lo que supone un método además seguro.

\section{CONCLUSIONES}

- Similar eficacia de la meperidina iv y la anestesia del plexo periprostático con lidocaína como analgesia en biopsias prostáticas.

- La introducción del transductor es más doloroso que la toma de muestras.

- La tolerancia al dolor no parece estar influida por la edad ni el nivel de estudios.

- La anestesia del plexo periprostático es un método seguro.

\section{REFERENCIAS}

1. Matlaga BR, Eskew LA, McCullough DL. Prostate biopsy: indications and tech nique. J Urol. 2003;169(1):12-19.

2. Aus G, Hermansson CG, Hugosson J, Pedersen KV. Transrectal ultrasound examination of the prostate: complications and acceptance by patients. $\mathrm{Br} \mathrm{J}$ Urol. 1993; 71(4): 457-459.

3. Peyromaure M, Ravery V, Messas A, Toublanc M, Boccon-Gibod L. Pain and morbidity of an extensive prostate 10-biopsy protocol: a prospective study in 289 patients. J Urol. 2002;167(1): 218-221.

4. Leibovici D, Zisman A, Siegel YI, Sella A, Kleinmann J, Lindner A.Local anesthesia for prostate biopsy by periprostatic lidocaine injection: a double-blind placebo controlled study. J Urol. 2002; 167(2 Pt 1):563-565. I

5. Nash P, Bruce J E, Indudhara R, and Shinohara K. Transrectal ultrasound guided prostatic nerve blochade eases systematic needle biopsy of the prostate. $\mathrm{J}$ Urol. 1996;155(2):607-609.

6. Bastide C, Lechevallier E, Eghazarian C, Ortega JC, Coulange C. Tolerante of pain during transrectal ultrasound-guided biopsy of the prostate: risk factors. Prostate Cancer Prostatic Dis. 2003; 6(3):239-341.

7. Rodríguez-Patrón R, Mayayo Dehesa T, Burgos Revilla FJ, Alonso González M, Garcia González R, Lennie Zucharino A. Tolerancia y complicaciones de la biopsia transrectal ecodirigida prostática ampliada a diez cilindros. Papel del bloqueo de haces neurovasculares con lidocaina. Arch Esp Urol. 2005;58(10):989-1001.

8. Rodriguez LV, Terris MK. Risks and complications of transrectal ultrasound guided prostate leedle biopsy: a prospective study and review of the literature. $J$ Urol 1998;160 (6 Pt 1):2115-2120.

9. Hergan L, Kashefi C, Parsons JK. Local anesthetic reduces pain associated with transrectal ultrasound-guided prostate biopsy: a meta-analysis. Urology. 2007 69(3):520-525.

10. Wu CL, Carter HB, Naqibuddin M, Fleisher LA. Effect of local anesthesics on patient recovery after transrectal biopsy. Urology. 2001;57(2):925-929.

11. Obek C, Ozkan B, Tunc B, Can G, Yalcin V, Solok V. Comparison of 3 different methods of anesthesia before transrectal prostate biopsy: a prospective randomized trial. J Urol. 2004;172(2):502-505.

12. Taverna G, Maffezini M, Benetti A, Seveso M, Giusti G and Graziotti P. A single injection of lidocaine as local anesthesia for ultrasound guided needle biopsy of the prostate. J Urol. 2002;167(1):222-223.

13. Issa MM, Bux S, Chun T, Petros JA, Labadia AJ, Anastasia K, Miller LE, Marshall FF.A randomized prospective trial of intrarectal lidocaine for pain control during transrectal prostate biopsy: the Emory University experience. J Urol. 2000;164(2):397-399.

14. Stirling BN, Shockley KF, Carothers GG et al. Comparison of local anesthesia techniques during transrectal ultrasound guided biopsies. Urology. 2002;60(1): 89-92.

15. Kaver I, Mabjeesh NJ, Matzkin H. Randomized prospective study of periprostatic local anestesia during transrectal ultrasound guided prostate biopsy. Urology. 2002;59(3):405-408. .

16. Obek C, Onal B, Ozkan B, Onder AU, Yalçin V, Solok V.. Is the periprostatic local anesthesia for transrectal ultrasound guided prostate biopsy associated with increased infectious or hemorrhagic complications? A prospective randomized trial. J Urol. 2002;168(2):558-561.

Correspondencia autor: Dr. S. Luján Marco

Servicio de Urología. Hospital Universitario La Fe

Avda. Campanar, 21 - 46009 Valencia. Tel.: 963862700

E-mail autor: slujanmarco@comv.es

Información artículo: Original - Cáncer de próstata

Trabajo recibido: octubre 2008

Trabajo aceptado: noviembre 2008 